PROCEEDINGS OF THE

AMERICAN MATHEMATICAL SOCIETY

Volume 126, Number 10, October 1998, Pages 2979-2986

S $0002-9939(98) 04633-4$

\title{
OPTIMAL CONTROL OF A FUNCTIONAL EQUATION ASSOCIATED WITH CLOSED RANGE SELFADJOINT OPERATORS
}

\author{
S. C. GAO AND N. H. PAVEL
}

(Communicated by Palle E. T. Jorgensen)

\begin{abstract}
Necessary and sufficient conditions for the optimality of a pair $\left(y^{*}, u^{*}\right)$ subject to $A y^{*}=B u^{*}+f$ are given. Here $A$ is a selfadjoint operator with closed range on a Hilbert space $\mathcal{H}$ and $B \in L(\mathcal{H})$. The case $B$ - unbounded is also discussed, which leads to some open problems. This general functional scheme includes most of the previous results on the optimal control of the $T$-periodic wave equation for all $T$ in a dense subset of $\mathbb{R}$. It also includes optimal control problems for some elliptic equations.
\end{abstract}

\section{INTRODUCTION}

This paper is concerned with the following optimal control problem

$$
\text { Minimize }: L(y, u)=G(y)+H(u)
$$

over all $(y, u)$ subject to

$$
A y=B u+f .
$$

Here $A$ is a selfadjoint operator $A: D(A) \subset \mathcal{H} \rightarrow R(A) \subset \mathcal{H}$ with closed range, where $\mathcal{H}$ is a real Hilbert space. $G$ and $H$ are lower-semicontinuous convex functionals from $\mathcal{H}$ into $(-\infty,+\infty]$ and satisfying Hypothesis (H1) in Section 2.

$B$ is supposed to be linear bounded one-to-one and onto from $\mathcal{H}$ into itself.

However, the case when $B$ is unbounded will be also discussed, as it is more important in applications and leads to delicate open problems.

The motivation of the problem $(\mathrm{P})$ consists in the fact that it includes the optimal control problems for the time-periodic wave equation. It is interesting in itself, too.

Received by the editors January 10, 1997 and, in revised form, February 27, 1997 and March $10,1997$.

1991 Mathematics Subject Classification. Primary 47N10, 47B25, 49K27.

Key words and phrases. Self-adjoint operators with closed range, optimal pairs, maximum principles, periodic waves.

The research of the first author was supported in part by the National Science Foundation of China.

The research of the second author was supported in part by the National Research Fund, Korean Research Foundation Project \#01-D0406 (jointly with Prof. J. K. Kim).

(C) 1998 American Mathematical Society 
As an example of $A$, consider the problem

$$
\begin{aligned}
y_{t t}-\Delta y & =f(t, x), \quad x \in \Omega, t \in(0, T), \\
y(t, x) & =0 \text { on }(0, T) \times \partial \Omega, \\
y(0, x) & =y(T, x) ; \quad y_{t}(0, x)=y_{t}(T, x), \quad x \in \Omega,
\end{aligned}
$$

where $\Omega$ is a bounded domain of $\mathbb{R}^{n}$ with a smooth boundary $\partial \Omega$ and $\Delta$ is the Laplace operator in the sense of distributions, $f \in L^{2}(Q)$.

Set $Q=(0, T) \times \Omega$ and

$$
\begin{aligned}
& C_{T}^{2}(\bar{Q})=\left\{\phi \in C^{2}(\bar{Q}) ; \phi(t, x)=0 \text { on }(0, T) \times \partial \Omega,\right. \\
& \quad \phi \text { satisfies the } T \text {-periodic conditions in }(1.2)\}, \\
& \qquad \mathcal{B} \phi=\phi_{t t}-\Delta \phi, \phi \in C_{T}^{2}(\bar{Q}) .
\end{aligned}
$$

Definition 1. $y \in L^{2}(Q)$ is said to be a weak (T-periodic) solution to the problem (1.2) if

$$
\int_{Q} y \mathcal{B} \bar{\phi} d t=\int_{Q} f \bar{\phi} d x, \forall \phi \in C_{T}^{2}(\bar{Q}) .
$$

Note that (1.4) can formally be obtained by multiplying by $\phi$, integrating by parts with respect to $t$ and using Green's formula

$$
\int_{\Omega}(\phi \Delta y-y \Delta \phi) d x=\int_{\partial \Omega}\left(\phi \frac{\partial y}{\partial \eta}-y \frac{\partial \phi}{\partial \eta}\right) d \sigma
$$

where $\frac{\partial \phi}{\partial \eta}$ is the outward derivative of $\phi$ on $\partial \Omega$.

Finally, set

$$
D(A)=\left\{y \in L^{2}(Q) ; \exists f \in L^{2}(Q) \text { such that (1.4) holds }\right\}
$$

and

$$
A y=f, y \in D(A) .
$$

In other words, $A y=f$ if and only if (1.4) holds. It was recently proved (cf. [6], [7]) that for $\Omega=(0, \pi) \times(0, \pi)$ and for every $T$ with $T^{2}=$ a rational multiple of $\pi^{2}$, the "weak solution operator" $A$ defined by $(1.6)+(1.7)$ satisfies the above conditions, i.e. it is selfadjoint in $\mathcal{H}=L^{2}$, with $R(A)$ closed. Moreover, $\operatorname{dim} N(A)=+\infty$.

Other examples of such operators can be found in ([1]-[2]). This example will be revisited at the end of the paper, in order to give an application of our main results. Another example of such $A$ is the elliptic operator $A y=\Delta y-a y$ with $D(A)=H_{0}^{1}(\Omega) \cap H^{2}(\Omega)$. Indeed, this operator is selfadjoint in $L^{2}(\Omega)$ with $R(A)=$ $L^{2}(\Omega)$, for all nonnegative numbers $a$.

\section{MAin RESUlts}

Let $\mathcal{H}$ be a real Hilbert space, $A: D(A) \subset \mathcal{H} \rightarrow \mathcal{H}$ a selfadjoint operator with closed range $R(A)$ and $B$ a continuous linear operator on $\mathcal{H}$. We formulate our problem as follows: For a given $f \in \mathcal{H}$, find a solution of

$$
\operatorname{Min}\left\{L(y, u) \mid(y, u) \in M_{f}\right\}
$$

where

$$
M_{f}=\{(y, u) \in \mathcal{H} \times \mathcal{H} \mid A y=B u+f, y \in D(A), u \in \mathcal{H}\}
$$

and $G$ and $H$ satisfy: 
(H1): $G$ and $H$ are lower semicontinuous convex functionals on $\mathcal{H}$ with

$$
\begin{gathered}
G(y) \geq a\|y\|^{2}+b, \text { for every } y \in \mathcal{H}, \\
H(u) \geq a_{1}\|u\|^{2}+b_{1}, \text { for every } u \in \mathcal{H},
\end{gathered}
$$

where $a, a_{1}>0, b, b_{1} \in \mathbb{R}$.

Remark 1. $M_{f}$ is weakly closed, i.e. if $\left(y_{n}, u_{n}\right) \in M_{f}, y_{n} \rightarrow y, u_{n} \rightarrow u$, then $(y, u) \in M_{f}$. This is because $B$ is bounded and $A$ is weakly closed in the following sense: $y_{n} \in D(A), y_{n} \rightarrow y$ and $A y_{n}$-bounded imply $y \in D(A)$ and $A y_{n} \rightarrow A y$.

Definition 2. We say that $(y, u) \in \mathcal{H} \times \mathcal{H}$ is an admissible pair if $(y, u) \in M_{f}$.

Definition 3. We say that $\left(y^{*}, u^{*}\right) \in \mathcal{H} \times \mathcal{H}$ is an optimal pair for the problem $(\mathrm{P})$ if and only if it is an admissible pair and it satisfies

$$
L\left(y^{*}, u^{*}\right)=\operatorname{Min}\left\{L(y, u) \mid(y, u) \in M_{f}\right\} .
$$

In this case, we also say that the problem $(\mathrm{P})$ admits an optimal pair.

We now state our main result.

Theorem 1. Suppose $A: D(A) \subset \mathcal{H} \rightarrow R(A) \subset \mathcal{H}$, with $R(A)$ closed, is selfadjoint; $B: \mathcal{H} \rightarrow \mathcal{H}$ is a linear continuous operator which is also one-to-one and onto. In addition, $(\mathrm{H} 1)$ holds and $\partial G$ and $\partial H$ are locally bounded at $\left(y^{*}, u^{*}\right)$ respectively. Then $\left(y^{*}, u^{*}\right)$ is an optimal pair of $(\mathrm{P})$ if and only if there exists $p^{*} \in D(A)$ such that

$$
A p^{*} \in-\partial G\left(y^{*}\right), \quad B^{*} p^{*} \in \partial H\left(u^{*}\right)
$$

where $\partial$ stands for the subdifferential operator.

Lemma 1. Suppose the conditions of Theorem 1 hold. Then problem (P) admits an optimal pair.

Proof. $B$ is onto, so $M_{f}$ in not empty. Denote

$$
d=\operatorname{Inf}\left\{L(y, u) \mid(y, u) \in M_{f}\right\} .
$$

Condition (H1) ensures that $d>-\infty$. Then there exists

$$
\left\{\left(y_{n}, u_{n}\right)\right\}_{n=1}^{\infty}, \quad\left(y_{n}, u_{n}\right) \in M_{f}
$$

for each $n \in \mathbb{N}$, such that

$$
d+1 \geq L\left(y_{n}, u_{n}\right) \geq a\left\|y_{n}\right\|^{2}+a_{1}\left\|u_{n}\right\|^{2}+e \text { for all } n \geq N \text { for some } N \in \mathbb{N} .
$$

(2.8) implies that both $\left\{y_{n}\right\}_{1}^{\infty}$ and $\left\{u_{n}\right\}_{1}^{\infty}$ are bounded and hence weakly convergent, say, $y_{n} \rightarrow y^{*}$ and $u_{n} \rightarrow u^{*}$ weakly in $\mathcal{H}$, as $n \rightarrow \infty$ (relabeling if necessary). Therefore

$$
A y_{n}=B u_{n}+f \rightarrow B u^{*}+f \text { weakly in } \mathcal{H} \text {, as } n \rightarrow \infty .
$$

Note that the graph of $A$ is weakly closed in $\mathcal{H} \times \mathcal{H}$, so (2.9), in conjunction with $y_{n} \rightarrow y^{*}$ weakly in $\mathcal{H}$, shows that $\left(y^{*}, u^{*}\right) \in M_{f}$, i.e.

$$
A y^{*}=B u^{*}+f \text {. }
$$

Therefore $\left(y^{*}, u^{*}\right)$ is an admissible pair. The lower semicontinuity of $L$ gives the following:

$$
L\left(y^{*}, u^{*}\right) \leq \varliminf_{n \rightarrow \infty} L\left(y_{n}, u_{n}\right)=d .
$$


On the other hand, $L\left(y^{*}, u^{*}\right) \geq d$. This, together with (2.11), shows that

$$
L\left(y^{*}, u^{*}\right)=\operatorname{Min}\left\{L(y, u) \mid(y, u) \in M_{f}\right\},
$$

i.e., the problem $(\mathrm{P})$ admits an optimal pair.

Lemma 2. Suppose that the conditions on $A$ and $B$ in Theorem 1 hold. In addition, suppose that $G$ and $H$ are Fréchet differentiable. Then the necessary condition for an admissible pair $\left(y^{*}, u^{*}\right)$ to be an optimal pair is the existence of an element $p^{*} \in D(A)$ such that

$$
A p^{*}=-\dot{G}\left(y^{*}\right), \quad B^{*} p^{*}=\dot{H}\left(u^{*}\right)
$$

where $\dot{G}$ is the Fréchet derivative of $G$.

Proof. One can show that the tangent space $T M_{f}\left(y^{*}, u^{*}\right)$ to $M_{f},\left(y^{*}, u^{*}\right) \in M_{f}$, or simply $T M_{f}$, is given by

$$
T M_{f}=\{(z, w) \in \mathcal{H} \times \mathcal{H} \mid A z=B w, z \in D(A), w \in \mathcal{H}\}
$$

independently of the point $\left(y^{*}, u^{*}\right) \in M_{f}$.

Now, if $\left(y^{*}, u^{*}\right)$ is optimal, we have

$$
\left(\dot{L}\left(y^{*}, u^{*}\right)\right)(z, w)=0, \text { for every }(z, w) \in T M_{f},
$$

$$
\left\langle\dot{G}\left(y^{*}\right), z\right\rangle+\left\langle\dot{H}\left(u^{*}\right), w\right\rangle=0, \text { for every }(z, w) \in T M_{f}
$$

where $\dot{G}\left(y^{*}\right)$ and $\dot{H}\left(u^{*}\right)$ are the Fréchet derivatives of the functionals $G$ and $H$ at $y^{*}$ and $u^{*}$, respectively. For $w=0$, i.e., for any $z \in N(A),(2.16)$ yields

$$
\left\langle\dot{G}\left(y^{*}\right), z\right\rangle=0 \text {, for every } z \in N(A) .
$$

This implies that $\dot{G}\left(y^{*}\right) \in R(A)$ since $\mathcal{H}$ admits the decomposition

$$
\mathcal{H}=R(A) \oplus N(A)
$$

under the conditions on $A$. Therefore there exists $\tilde{p}^{*} \in D(A)$ such that

$$
A \tilde{p}^{*}=\dot{G}\left(y^{*}\right) .
$$

Thus (2.16) becomes

$$
\left\langle A \tilde{p}^{*}, z\right\rangle+\left\langle\dot{H}\left(u^{*}\right), w\right\rangle=0, \text { for every }(z, w) \in T M_{f}
$$

or equivalently (as $B^{-1}$ exists)

$$
\left\langle\tilde{p}^{*}, A z\right\rangle+\left\langle\left(B^{*}\right)^{-1} \dot{H}\left(u^{*}\right), B w\right\rangle=0, \text { for every }(z, w) \in T M_{f}
$$

i.e.,

$$
\left\langle\tilde{p}^{*}+\left(B^{*}\right)^{-1} \dot{H}\left(u^{*}\right), A z\right\rangle=0, \text { for every } z \in D(A)
$$

as $R(B)=\mathcal{H}$. Indeed, for every $z \in D(A)$, there is $w$ such that $A z=B w$, as $B$ is onto. Consequently, one has

$$
\tilde{p}^{*}+\left(B^{*}\right)^{-1} \dot{H}\left(u^{*}\right) \in N(A)
$$

again because of the decomposition $(2.18)$ of $\mathcal{H}$. Denote

$$
\tilde{p}^{*}+\left(B^{*}\right)^{-1} \dot{H}\left(u^{*}\right)=\eta \in N(A), \text { and } p^{*}=\eta-\tilde{p}^{*} .
$$


Clearly (2.24) implies (2.13), which completes the proof. Note that Conditions (2.13) may not be sufficient, due to the fact that in Lemma 2, $G$ and $H$ are not supposed to be convex so they may not be subdifferentiable. When they are, (2.13) are sufficient, too(see (2.38)).

\section{Proof of Theorem 1. Necessity.}

Step 1. If $G$ and $H$ are Fréchet differentiable, then by Lemma 2, there is $p^{*} \in D(A)$ such that equalities in (2.13) hold. In this case (2.13) and (2.5) are equivalent as $\partial G=\dot{G}$ and $\partial H=\dot{H}$.

Step 2. Suppose $G$ and $H$ are merely lower semicontinuous and $\partial G$ and $\partial H$ are locally bounded at $y^{*}$ and $u^{*}$, respectively. We can use the convex approximation scheme $L_{\lambda}$ of $L$, i.e., consider the following optimal problem of Barbu's type:

$\left(P_{\lambda}\right) \quad$ Minimize $: L_{\lambda}(y, u)=G_{\lambda}(y)+H_{\lambda}(u)+\frac{1}{2}\left\|y-y^{*}\right\|^{2}+\frac{1}{2}\left\|u-u^{*}\right\|^{2}$

over $M_{f}$, where $G_{\lambda}$ and $H_{\lambda}$ are the convex regularizations of $G$ and $H$, respectively, i.e.,

$$
G_{\lambda}(y)=\operatorname{Min}\left\{G(x)+\frac{1}{2 \lambda}\|y-x\|^{2} \mid\right\}, \lambda>0, y \in D(G) .
$$

Here $G_{\lambda}$ and $H_{\lambda}$ are Fréchet differentiable, and $\dot{G}_{\lambda}(y)=(\partial G)_{\lambda}(y) \in \partial G\left(J_{\lambda}^{G} y\right)$, $J_{\lambda}^{G}=(I-\lambda \partial G)^{-1}$ (see e.g. [8], Appendix, on these Yosida operators $J_{\lambda}$ and $A_{\lambda}$, and convex regularizations). On the basis of Step 1, there exists an optimal pair $\left(y_{\lambda}^{*}, u_{\lambda}^{*}\right)$ for $\left(P_{\lambda}\right)$ and, correspondingly, $p_{\lambda}^{*} \in D(A)$ such that

$$
A p_{\lambda}^{*}=-\dot{G}_{\lambda}\left(y_{\lambda}^{*}\right)-\left(y_{\lambda}^{*}-y^{*}\right), B^{*} p_{\lambda}^{*}=\dot{H}_{\lambda}\left(u_{\lambda}^{*}\right)+\left(u_{\lambda}^{*}-u^{*}\right) .
$$

Equalities

$$
G_{\lambda}\left(y_{\lambda}^{*}\right)=G\left(J_{\lambda} y_{\lambda}^{*}\right)+\frac{\lambda}{2}\left\|\dot{G}_{\lambda}\left(y_{\lambda}^{*}\right)\right\|^{2}, \quad J_{\lambda}=J_{\lambda}^{G}
$$

and

$$
J_{\lambda}\left(y_{\lambda}^{*}\right)+\lambda \dot{G}_{\lambda}\left(y_{\lambda}^{*}\right)=y_{\lambda}^{*}
$$

together with the inequalities

$$
G_{\lambda}\left(y_{\lambda}^{*}\right) \geq a\left\|J_{\lambda} y_{\lambda}^{*}\right\|^{2}+\frac{\lambda}{2}\left\|\dot{G}_{\lambda}\left(y_{\lambda}^{*}\right)\right\|^{2}+b
$$

and

$$
\begin{aligned}
G\left(J_{\lambda}^{G} y_{\lambda}^{*}\right)+H\left(J_{\lambda}^{H} u_{\lambda}^{*}\right) & \leq G_{\lambda}\left(y_{\lambda}^{*}\right)+H_{\lambda}\left(u_{\lambda}^{*}\right) \\
& \leq L_{\lambda}\left(y_{\lambda}^{*}, u_{\lambda}^{*}\right) \leq L_{\lambda}\left(y^{*}, u^{*}\right) \\
& =G_{\lambda}\left(y^{*}\right)+H_{\lambda}\left(u^{*}\right) \leq L\left(y^{*}, u^{*}\right)
\end{aligned}
$$

imply that $\sqrt{\lambda}\left\|\dot{G}_{\lambda}\left(y_{\lambda}^{*}\right)\right\|$ and $\left\|J_{\lambda}\left(y_{\lambda}^{*}\right)\right\|$ are bounded, so by (2.29) $y_{\lambda}^{*}$ is bounded, too. Say $y_{\lambda}^{*} \rightarrow \tilde{y}^{*}$ weakly in $\mathcal{H}$ as $\lambda \rightarrow 0$ for some $\tilde{y}^{*} \in \mathcal{H}$. Then by $(2.29), J_{\lambda}\left(y_{\lambda}^{*}\right) \rightarrow \tilde{y}^{*}$. Similarly, $u_{\lambda} \rightarrow \tilde{u}^{*}$ weakly in $\mathcal{H}$ as $\lambda \rightarrow 0$ for some $\tilde{u}^{*} \in \mathcal{H}$. The same argument used in the proof of Lemma 1 can be used here to show that $\left(\tilde{y}^{*}, \tilde{u}^{*}\right) \in M_{f}$. 
The above inequality, together with the semicontinuity of $G$ and $H$, implies that

$$
\begin{aligned}
L\left(y^{*}, u^{*}\right) & \leq L\left(\tilde{y}^{*}, \tilde{u}^{*}\right) \\
& \leq \lim _{\lambda \rightarrow 0}\left(G_{\lambda}\left(y^{*}\right)+H_{\lambda}\left(u^{*}\right)\right) \\
& \leq \lim _{\lambda \rightarrow 0} L_{\lambda}\left(y_{\lambda}^{*}, u_{\lambda}^{*}\right) \\
& \leq L\left(y^{*}, u^{*}\right) ;
\end{aligned}
$$

therefore

$$
\lim _{\lambda \rightarrow 0} L_{\lambda}\left(y_{\lambda}^{*}, u_{\lambda}^{*}\right)=\lim _{\lambda \rightarrow 0}\left(G_{\lambda}\left(y_{\lambda}^{*}\right)+H_{\lambda}\left(u_{\lambda}^{*}\right)\right)=L\left(y^{*}, u^{*}\right) .
$$

This yields $\lim _{\lambda \rightarrow 0}\left\|y_{\lambda}^{*}-y^{*}\right\|=\lim _{\lambda \rightarrow 0}\left\|u_{\lambda}^{*}-u^{*}\right\|=0$ and consequently, $\tilde{y}^{*}=y^{*}$ and $\tilde{u}^{*}=u^{*}$.

We now rely on the facts:

$$
\begin{gathered}
\dot{G}_{\lambda}\left(y_{\lambda}^{*}\right) \in \partial G\left(J_{\lambda}^{G} y_{\lambda}^{*}\right), \quad \dot{H}_{\lambda}\left(u_{\lambda}^{*}\right) \in \partial H\left(J_{\lambda}^{H} u_{\lambda}^{*}\right), \\
J_{\lambda}^{G}\left(y_{\lambda}^{*}\right) \rightarrow y^{*}, \quad J_{\lambda}^{H}\left(u_{\lambda}^{*}\right) \rightarrow u^{*} \text { strongly. }
\end{gathered}
$$

Note that (2.35) follows from (2.29) in conjunction with $y_{\lambda}^{*} \rightarrow y^{*}$, due to

$$
\left\|J_{\lambda}^{G}\left(y_{\lambda}^{*}\right)-y_{\lambda}^{*}\right\| \leq \sqrt{\lambda}\left(\sqrt{\lambda}\left\|\dot{G}_{\lambda}\left(y_{\lambda}^{*}\right)\right\|\right) .
$$

As $\partial G$ and $\partial H$ are locally bounded at $y^{*}$ and $u^{*}$, respectively, it follows that $\dot{G}_{\lambda}\left(y_{\lambda}^{*}\right)$ and $\dot{H}_{\lambda}\left(u_{\lambda}^{*}\right)$ are bounded, so we may assume $\dot{G}_{\lambda}\left(y_{\lambda}^{*}\right) \rightarrow \alpha$ and $\dot{H}_{\lambda}\left(u_{\lambda}^{*}\right) \rightarrow \beta$ weakly.

As $\partial G$ and $\partial H$ are maximal monotone, we can pass to limit in (2.34) for $\lambda \rightarrow 0$, to get $\alpha \in \partial G\left(y^{*}\right), \beta \in \partial H\left(u^{*}\right)$.

From (2.27) it follows that $p_{\lambda}^{*}$ is bounded. Say $p_{\lambda}^{*} \rightarrow p^{*}$. Letting $\lambda \rightarrow 0$ in $(2.27)$ we get

$$
A p^{*}=-\alpha \in-\partial G\left(y^{*}\right), \quad B^{*} p^{*}=\beta \in \partial H\left(u^{*}\right)
$$

which completes the proof.

Sufficiency. For any $(y, u) \in M_{f}$, we have

$$
\begin{aligned}
L(y, u)-L\left(y^{*}, u^{*}\right) & =G(y)-G\left(y^{*}\right)+H(u)-H\left(u^{*}\right) \\
& \geq\left\langle-A p^{*}, y-y^{*}\right\rangle+\left\langle B^{*} p^{*}, u-u^{*}\right\rangle \\
& =-\left\langle p^{*}, A\left(y-y^{*}\right)\right\rangle+\left\langle p^{*}, B\left(u-u^{*}\right)\right\rangle \\
& =0
\end{aligned}
$$

which completes the proof.

In the case in which $B$ is not one-to-one, the following result holds:

Theorem 2. Let $A$ and $B$ be selfadjoint operators in $\mathcal{H}$ with $R(A)$ and $R(B)$ closed in $\mathcal{H}$ and $R(A) \subset R(B)$. Let $G$ and $H$ be Frechét differentiable, satisfying (H1). Then a necessary and sufficient condition for an admissible pair $\left(y^{*}, u^{*}\right)$ to be optimal is the existence of an element $p^{*} \in D(A)$ such that

$$
A p^{*}=-\dot{G}\left(y^{*}\right) ; B p^{*}=\dot{H}\left(u^{*}\right) .
$$

Proof. In this case $T M_{f}\left(y^{*}, u^{*}\right) \supset S$ with

$$
S=\{(z, w) ; \quad z \in D(A), w \in D(B), A z=B w\} .
$$


Indeed, $\left(y^{*}, u^{*}\right)+\lambda(z, w) \in M_{f}, \forall \lambda \in \mathbb{R}$. Arguing as in the proof of Lemma 2, we have

$$
\left\langle\dot{G}\left(y^{*}\right), z\right\rangle+\left\langle\dot{H}\left(u^{*}\right), w\right\rangle=0, \quad \forall(z, w) \in S
$$

which yields (2.19).

For $z=0,(2.41)$ implies $\left\langle\dot{H}\left(u^{*}\right), w\right\rangle=0 \forall B w=0$ so due to (2.18) we have

$$
\dot{H}\left(u^{*}\right) \in R(B) \text {. }
$$

But $B$ is invertible on $R(B)$ so in view of (2.41), (2.21) makes sense for $(z, w) \in S$. Therefore, according to (2.19)-(2.24) the conclusion of Theorem 2 holds.

We now can briefly sketch an application of our general results.

Let $g: \mathbb{R} \rightarrow \mathbb{R}$ and $h: \mathbb{R} \rightarrow \mathbb{R}$ be lower semicontinuous convex functions such that their $L^{2}$ convex integrands $G$ and $H$ satisfy the Hypothesis (H1). Recall that $G$ is precisely defined by $G y=\int_{Q} g(y(t, x)) d t d x$.

Let $\phi$ be such that the operator $B,(B u)(t, x)=\phi(x) u(t, x)$ is one-to-one and onto in $L^{2}(Q)$. Then the problem

$$
\text { Minimize: } \int_{Q}(g(y(t, x))+h(u(t, x))) d t d x
$$

subject to

$$
y_{t t}-\Delta_{x} y=\phi(x) u(t, x)+f(t, x), x \in \Omega, t \in(0, T), \quad \Omega=(0, \pi) \times(0, \pi),
$$

$$
\begin{aligned}
& y(t, x)=0 \text { on } \partial((0, \pi) \times(0, \pi)), \quad t \in(0, T), \\
& y(0, x)=y(T, x) ; y_{t}(0, x)=y_{t}(T, x),
\end{aligned}
$$

where $T^{2}=$ a rational multiple of $\pi^{2}$ as indicated in Section 1, has a solution (optimal pair) $\left(y^{*}, u^{*}\right)$. Moreover, a pair $\left(y^{*}, u^{*}\right)$ is optimal iff there is $p^{*}$ such that

$$
\begin{aligned}
p_{t t}^{*}-\Delta p^{*} & =-w(t, x), \\
p^{*}(t, x) & =0 \text { on } \partial((0, \pi) \times(0, \pi)), \\
p^{*}(0, x) & =p^{*}(T, x) ; p_{t}^{*}(0, x)=p_{t}^{*}(T, x), \\
w(t, x) & \in \partial g\left(y^{*}(t, x)\right), \\
\phi(x) p^{*}(t, x) & \in \partial h\left(u^{*}(t, x)\right) \text { a.e. in } Q,
\end{aligned}
$$

or equivalently

$$
u^{*}(t, x) \in \partial h^{*}\left(\phi(x) p^{*}(t, x)\right) \text { a.e. in } Q
$$

where $h^{*}$ is the conjugate of $h$, i.e.

$$
h^{*}(r)=\sup \{r v-h(v) ; \quad v \in \mathbb{R}\}, r \in \mathbb{R} .
$$

If $\phi: \Omega \rightarrow \mathbb{R}$ is a measurable function satisfying

$$
0<a \leq \phi(x) \leq b<\infty, \text { a.e. in } \Omega,
$$

then the operator $B$ given by

$$
(B u)(t, x)=\phi(x) u(t, x), \text { a.e. in } Q
$$

is one-to-one and onto in $L^{2}(Q)$ so the above results hold. 
If instead of (2.48) $\phi$ satisfies only

$$
0 \leq \phi(x) \text {, a.e. in } \Omega \text {, }
$$

then $B$ is (unbounded) selfadjoint operator in $L^{2}(Q)$.

Indeed, $B$ is monotone (i.e. $\langle B u, u\rangle \geq 0, \forall u \in D(B)$ ), $R(I+B)=L^{2}(Q)$, so $B$ is maximal monotone. On the other hand $B$ is also symmetric, or a symmetric maximal monotone operator in a Hilbert space is selfadjoint, as claimed.

It follows that if

$$
0<a \leq \phi(x),
$$

then the results above on the optimal pair hold.

Indeed, (2.51) implies that $N(B)=0$, so $R(B)=L^{2}(Q) \supset R(A)$ and $B$ is selfadjoint with closed range.

Details and other examples, as well as the case $B$-nonlinear will be studied elsewhere. The existence of optimal pairs in the case in which $B$ is unbounded, remains an open problem.

\section{ACKNOWLEDGEMENTS}

We thank the referee for some useful suggestions, which have contributed to the improvement of this paper.

\section{REFERENCES}

1. V. Barbu, Optimal control of the one dimensional periodic wave equation, Appl. Math. Optimiz., 35(1997), 77-90. CMP 97:03

2. V. Barbu and N. H. Pavel, Periodic solutions to nonlinear 1-D wave equation with $x$-dependent coefficients, Trans. Amer. Math. Soc 349 (1997), no. 5, 2035-2048. MR 97h:35129

3. H. Brezis, Periodic solutions of nonlinear vibrating string and duality principles, Bull. AMS, 8(1983), 409-426. MR 84e:35010

4. J. K. Kim and N. H. Pavel, Optimal control of the periodic wave equation, Proceedings of Dynamical Sytems and Applications. Vol.2(1996),09-14, Atlanta, Georgia, May 1995 MR 97j:49042

5. $-L^{\infty}$-optimal control of the $1-D$ wave equation with $x$-dependent coefficients, Nonlinear Analysis, TMA (to appear).

6. __ Existence and regularity of weak periodic solutions of the 2-D wave equation, Nonlinear Analysis, TMA (to appear).

7. N. H. Pavel, Periodic solutions to nonlinear 2-D wave equations, Lecture Notes in Pure and Applied Mathematics, Marcel Dekker, Vol.178(1996), 243-250 MR 97c:35138

8. __ Nonlinear Evolution Operators and Semigroups. Applications to Partial Differential Equations, Lecture Notes in Pure and Applied Mathematics, Springer-Verlag, Vol 1260(1987). MR 88j: 47087

Department of Mathematics, Ohio University, Athens, Ohio 45701

E-mail address: shugao@bing.math.ohiou.edu

E-mail address: npavel@bing.math.ohiou.edu 